\title{
Analysis of NASA Common Research Model Dynamic Data
}

\author{
S. Balakrishna ${ }^{1}$ \\ Vigyan Inc, Hampton, Va, 23666 \\ Michael J Acheson ${ }^{2}$ \\ Project manager, NFMTC, LaRC, Hampton, Va, 23681
}

\begin{abstract}
Recent NASA Common Research Model (CRM) tests at the Langley National Transonic Facility (NTF) and Ames 11-foot Transonic Wind Tunnel (11-foot TWT) have generated an experimental database for CFD code validation. The database consists of force and moment, surface pressures and wideband wing-root dynamic strain/wing Kulite data from continuous sweep pitch polars. The dynamic data sets, acquired at $12,800 \mathrm{~Hz}$ sampling rate, are analyzed in this study to evaluate CRM wing buffet onset and potential CRM wing flow separation.
\end{abstract}

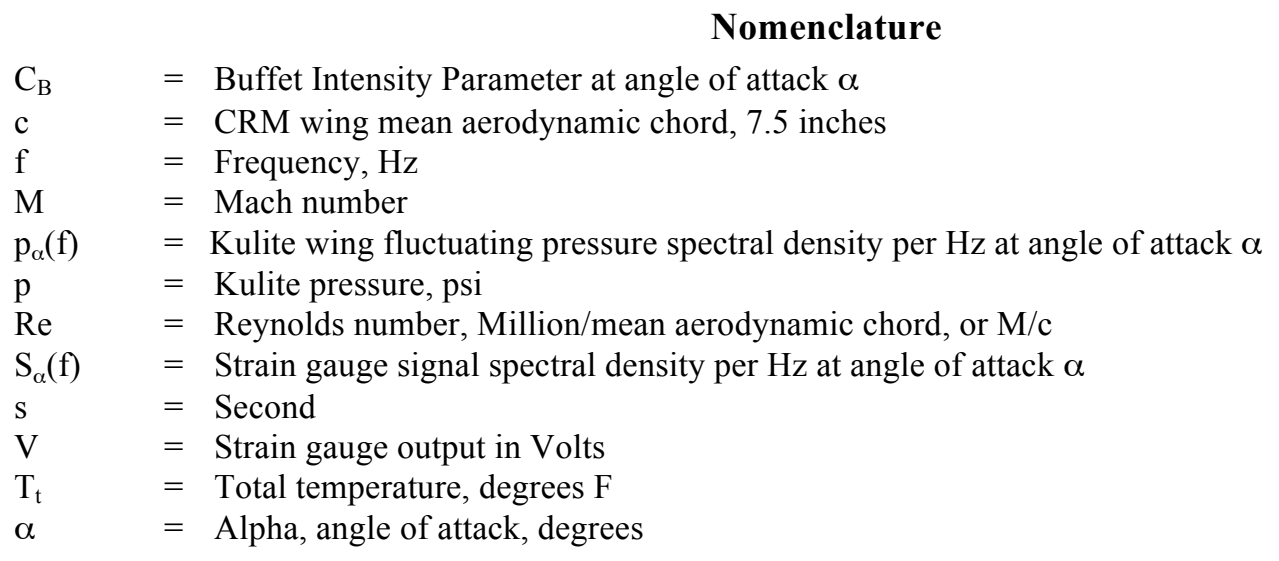

\section{Introduction}

$\mathrm{B}$ uffet onset in an aircraft is the beginning of the structural response to an unsteady aerodynamic excitation from air flow over the wing and body. Buffet is related to flow separation that may occur over wings at high lift, due to shock-boundary layer interaction and is a dynamic phenomenon associated with wide frequency band pressure fluctuations driving the wing structure. Wind tunnel models in transonic flows experience structural response from sting-model dynamics and tunnel flow turbulence. Therefore, buffet onset of a wind tunnel model is determined as the angle of attack at which wing flow induced aerodynamic excitation initiates a structural response, over and above those created by tunnel turbulence and sting dynamics. In order to experimentally identify the initiation of flow separation and its structural excitation of wings, wideband wing-root strain gauge sensors are used to measure dynamic bending moment on a wind tunnel model. Such a sensor will be able to pickup flow separation dynamics anywhere along the wing globally. If the physical location of the wing surface where flow separation is likely to occur is known apriori, it is possible to mount dynamic pressure sensors (Kulites) in that area. However, these Kulites can provide only local unsteady pressure information. The Common Research Model (CRM) is an open geometry, generic transport model developed by NASA for testing across multiple wind tunnels to support Computational Fluid Dynamics (CFD) code validation in transonic flows. CRM wind tunnel testing involved studying static and dynamic force, moment, and pressure data while exposed to flows duplicating elastic-inertiaviscosity similitude typical of full-scale flight. Dedicated, slow continuous-pitch runs, reaching angles of attack where potential flow separation over the wing was likely, were made. Data acquired consisted of dynamic wing-

\footnotetext{
${ }^{1}$ Senior Research Scientist, Vigyan Inc.

${ }^{2}$ Group leader, Technologies Application Branch, MS 237, NASA Langley Research Center.
} 
root strain and dynamic pressure (Kulite) data at $\mathrm{M}=0.70$ and 0.85 for three different Reynolds numbers of $5,19.8$ and $30 \mathrm{M} / \mathrm{c}$, with data sampled at $12,800 \mathrm{~Hz}$ at NTF. Tests were then repeated in the 11 -foot $\mathrm{TWT}$ at $\mathrm{Re}=5 \mathrm{M} / \mathrm{c}$. This paper presents analysis of the wing-root strain and dynamic pressure data with a view to relate information to potential flow separation and onset of buffet on CRM.

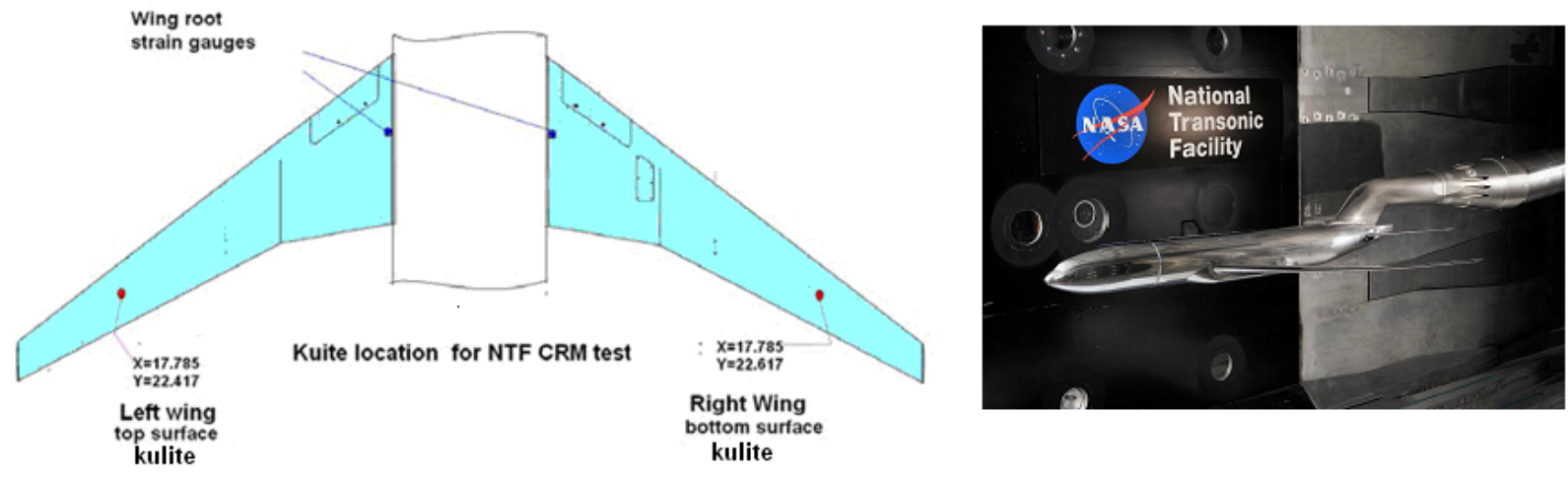

Figure 1: CRM Model showing dynamic sensors

\section{NASA Common Research Model and Wing Dynamics Sensors}

The Common Research Model is a generic transport model instrumented for force and moment measurements, wing pressures, wing-root strains and dynamic pressures. The model has wing mean aerodynamic chord of 7.5 inches and a wing area of $3.01 \mathrm{ft}^{2}$ and is mounted on an upper swept sting with clean base flow. The CRM was mounted on the NTF118 balance.

Figure 1 shows the location of dynamic pressure sensors on CRM model. Kulites are located at about $70 \%$ of span. The left-hand wing has sensors located on top surface while the right-hand wing has its sensors on bottom surface. Wing-root strain is measured using half bridges with a 5-volt bridge excitation.. Kulites signals are amplified to provide about $6.3 \mathrm{psi} /$ volt. Kulite sensor locations were based on expectations of flow separation from CFD analysis.

Wing-root strain gauges provide root bending moment data. Bending moment is the integral of the product of local pressure forces times the local distance. If the wing loading has components of flow separation at any location with its inherent wide-band pressure signature, the strain gauges at the wing-root can see this signature as a dynamic bending moment. By analyzing the strain gauge signal, one can infer the onset of flow separation as a distinct change in root-mean-squared ( $r m s$ ) amplitude. The $r m s$ value of wing-root strain gauge signal thus provides the magnitude of the dynamic pressure signature of flow separation. The Kulites provide fluctuating pressure signatures specific to its location and will respond only if the flow separation occurs at that point. Wind tunnel balance data is usually heavily filtered and these channels can tolerate electrical noise. However, in case of dynamic sensors, all the buffet information is in higher frequencies and not at static mean levels. Hence, the need for high signal-to-noise ratio is imperative for dynamic sensors. NTF and 11-foot TWT strain gauge and Kulite signals during tests had different signal-to-noise ratios, which potentially impacted the analysis as will be discussed later.

\section{Buffet Intensity Measure}

A literature survey of the determination of buffet from wind tunnel model tests with wing-root strain gauges ${ }^{1-3}$ shows that a buffet intensity or buffet coefficient $\mathrm{C}_{\mathrm{B}}$ is determined based on the area of the wing-root strain spectrum at a given angle of attack $(\alpha)$, for a given flow condition. Different algorithms are used for normalizing data such as geometry of the wing, dynamic pressure, and lift coefficient. Reference 1 used an aeroelastic flutter 
approach using the inverse amplitude of the spectral content. Mabey ${ }^{2}$ used the area under spectral response of wingroot gauges normalized to the dynamic pressure. In all cases, wide-band strain wing-root bending moment and its change relative to angle of attack appears to be the basis to determine the onset of buffet.

For purposes of analyzing the CRM wing-root strain dynamic data work, we use a simple parameter based on growth of wing-root bending moment energy during a wind tunnel test polar. It is assumed that, at zero angle of attack flow over the wing is clean and it has no areas of flow separation. Hence this condition contains the base response of CRM to wind tunnel turbulence and other mechanical dynamics at a given flow condition. As the angle of attack is increased, any growth of dynamic strain gauge response can be attributed to development of flow separation as the wing develops higher lift. The following buffet intensity measure is used in this analysis. It is clearly a function of angle of attack.

$$
C_{B}(\alpha)=\frac{\int_{0}^{f M A X} S_{\alpha}(f) d f}{\int_{0}^{f M A X} S_{0}(f) d f}
$$

Dynamic tests on CRM were conducted in a slow polar sweep from near zero angle of attack, while recording

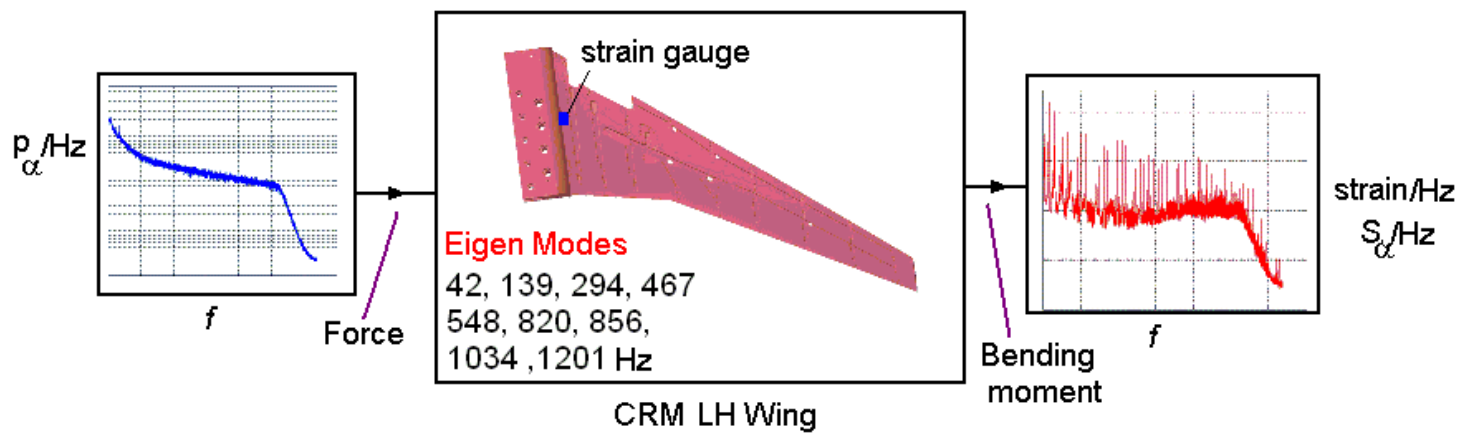

Figure 2: Wing root excitation

the strain gauge signals sampled at $12,800 \mathrm{~Hz}$ (in one case to $25600 \mathrm{~Hz}$ ). The quantity fMAX was defined as the Nyquist frequency, which is half the sampling rate. This Buffet Intensity Parameter provides growth of wing-root bending moment strain energy as a function of $\alpha$ due to vibrations caused by any flow separation over the wings.

Force excitation on the CRM wing is due to steady-state lift aerodynamics with unsteady forces from tunnel flow turbulence, and flow separation on the wing, if any, as shown in figure 2. Fluctuating pressures in the tunnel and flow separation are known to have bandwidth to many kilohertz. The CRM left wing first structural bending mode is at $42 \mathrm{~Hz}$ followed by nine other modes up to $1200 \mathrm{~Hz}$. The wide-band fluctuating pressure forces acting on the wing surface create a wide-band bending-moment response in the wing-root. For the CRM test, initially $12,800 \mathrm{~Hz}$ was chosen as sampling frequency with a Nyquist frequency of $6400 \mathrm{~Hz}$. This was based on wing structural Eigen modes ranging from 42 to $1200 \mathrm{~Hz}$, up to which wing could respond structurally. To confirm the adequacy of this bandwidth, one repeat polar sweep was sampled at $25600 \mathrm{~Hz}$. Dynamic tests were performed on the baseline WingBody (WB) configuration of CRM. Trip dots were used for $R e=5 \mathrm{M} / \mathrm{c}$ testing, while $\mathrm{Re}=19.8$ and $30 \mathrm{M} / \mathrm{c}$ tests did not utilize trip dots.

\section{Signal spectrum}

All data analyses were performed in MATLAB $\odot$. A custom algorithm was developed to determine the amplitude spectrum of strain gauge, Kulite and angle of attack signals in successive, and typically, one-quarter to one-second windows for the full polar length. This rectangular windowing has no effect on spectra as there are 3,200 or 12,800 samples in the window and there are very little round off errors. During NTF dynamic tests, polar sweeps used a 
pitch a rate of 0.1 degrees/s. Hence, all the one second window spectral data analyzed would each be associated with mean angle of attack to $+/-0.05$ degrees accuracy or better. The algorithm uses a Fast Fourier Transform and truncates it appropriately to arrive at signal amplitude resolved to frequency, providing signal mean and standard deviation. This algorithm was applied to strain signals, Kulite signals and angle of attack signals so that relative spectral amplitudes would be time synchronized relative to each other. In each window, the signals were integrated to arrive at $\mathrm{S}_{\alpha}, \mathrm{P}_{\alpha}$ and average angle of attack so that spectra can be analyzed as functions of angle of attack. No digital filtration of signals was used in the analysis. Further, the amplitude of wing-root strain signal was normalized to base signal level since signal engineering units have no direct usefulness. However, Kulite data was scaled to pressure units.

\section{CRM Wing-root strain and wing buffet onset from NTF tests}

NTF runs were performed in a slow, continuous sweep of $\alpha$ from about 0.5 degrees up to 8 degrees for low Reynolds number runs, and up to 5 degrees for high Reynolds number runs. Pitch rate was 0.1 degrees/s in forward sweep and 0.8 degrees/s when returning back to $\alpha=0^{\circ}$. In Fig. 3 the first plot shows the raw wing-root strain gauge signal with time, and second plot shows the average wing-root strain spectrum for $\mathrm{M}=0.85$ run at $5 \mathrm{M} / \mathrm{c}$ Reynolds number polar sweep. The spectrum is the average for the whole polar, and has many spikes, which are $60 \mathrm{~Hz}$ electrical noise and its harmonics. The spectrum amplitude in $\mathrm{V} / \mathrm{Hz}$ shows the various modal structural responses of
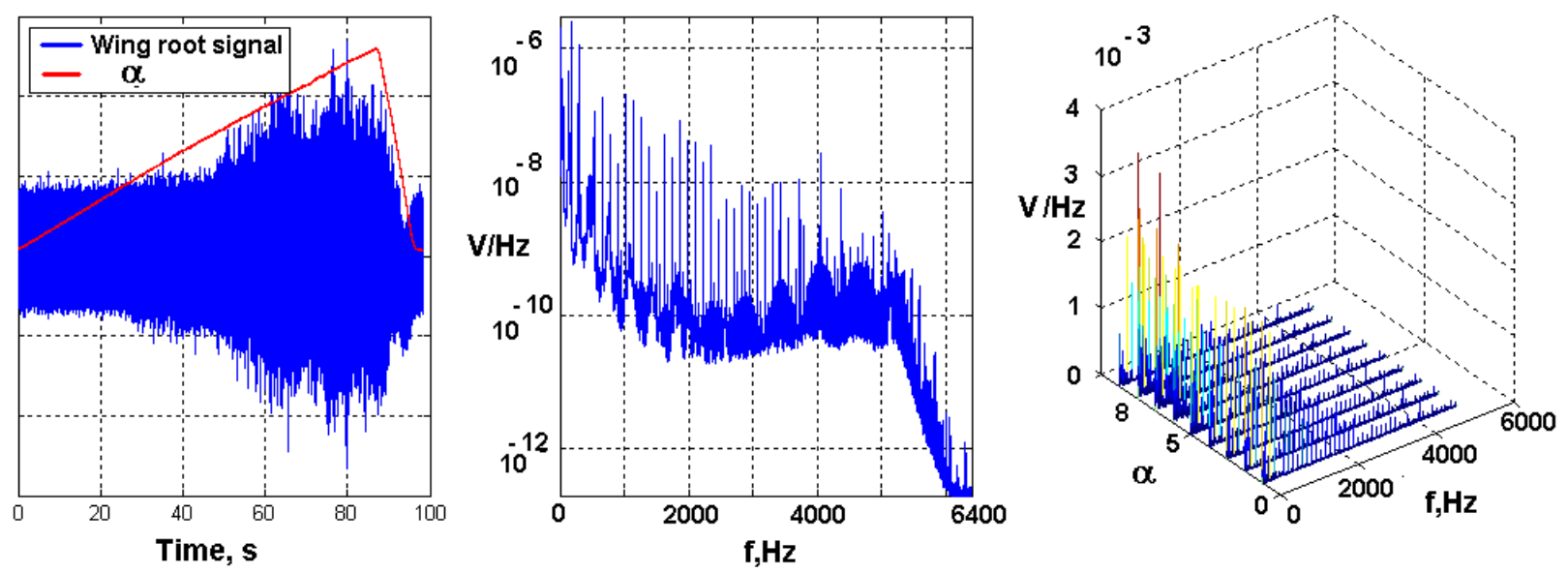

Figure 3: Wing root Spectra from NTF Dynamic test at $M=0.85, R e=5 M / c, T_{t}=120 F$

the wing up to $6400 \mathrm{~Hz}$. There are many modes visible in basic spectrum if the $60 \mathrm{~Hz}$ and its aliases are ignored. The waterfall plot on right shows evolution of spectra as a function of angle of attack clearly showing that wing-root response varies with angle of attack and wing lift.

It is usual to provide buffet intensity as a function of lift coefficient. In this work, data is provided as a function of angle of attack, which is related to lift. Figure 4, plot 1 illustrates left-wing (top) buffet intensity parameter $C_{B}$ for Mach numbers of $\mathrm{M}=0.7$ and 0.85 at a Reynolds number of $5 \mathrm{M} / \mathrm{c}$. Trip dots were used for these polars. It can be seen that buffet onset occurs at 3 degrees angle of attack at $\mathrm{M}=0.85$. At $\mathrm{M}=0.7$ there is no evidence of buffet till about 5-6 degrees. Buffet gets more severe at about 5 to 6 degrees angle of attack for $\mathrm{M}=0.85$ case. Figure 4 , plot 2 shows that sampling rate/ bandwidth increase from $12.8 \mathrm{kHz}$ to $25.6 \mathrm{kHz}$ does not change the buffet picture significantly, suggesting that all wing structural dynamic response due to flow separation process occur at frequencies lower than $6400 \mathrm{~Hz}$. 

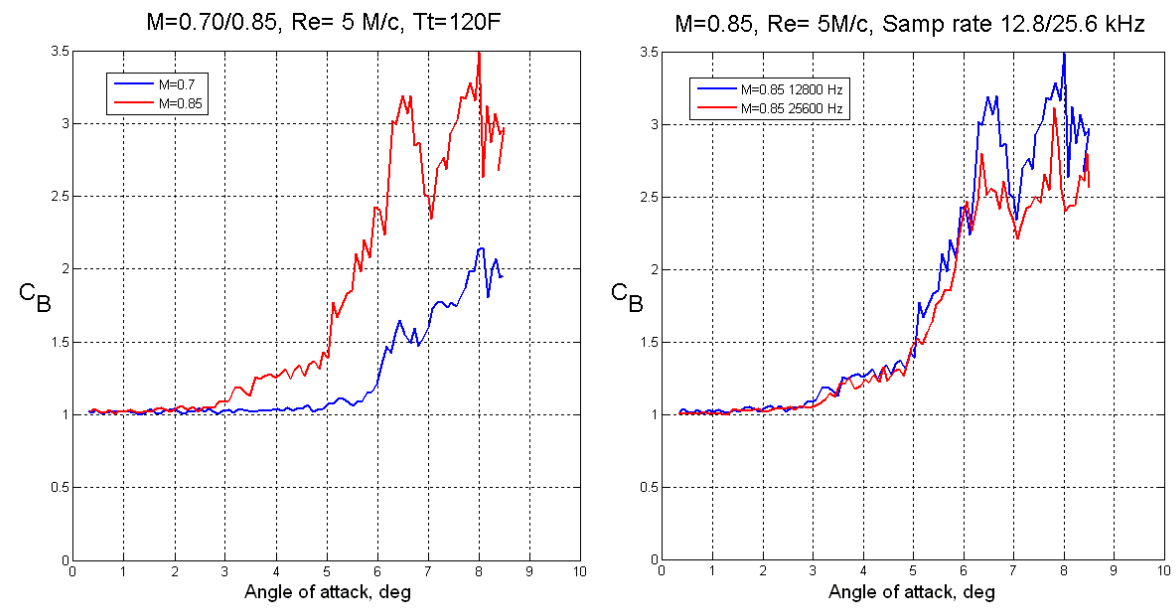

Figure 4: Buffet intensity at $5 \mathrm{M} / \mathrm{c}$ Reynolds number and $M=0.70$ and 0.85 , and with sampling rate change Wing Body configuration with trip dots

Figure 5, Plot 1 shows data for left wing (top) and buffet intensity parameter $\mathrm{C}_{\mathrm{B}}$ for 1 to 5 degree polars at $\mathrm{M}=0.7$ and $\mathrm{M}=0.85$ at Reynolds number of $19.8 \mathrm{M} / \mathrm{c}$. This pair of polars was performed at a cryogenic temperature of $-182 \mathrm{~F}$ using 0.1 degrees/s pitch rate. Trip dots were not used in these polars. Again, the onset of buffet is indicated at an angle of attack of 3 degrees for $M=0.85$. No buffet is indicated at $M=0.7$. Figure 5, plot 2 shows another pair of $\mathrm{M}=0.7$ and 0.85 runs. This pair of polars was performed at a cryogenic temperature of $-250^{\circ} \mathrm{F}$ using 0.1 degrees/s pitch rate, at a Reynolds number of $30 \mathrm{M} / \mathrm{c}$. Again, onset of buffet occurs at about 3 degrees angle of attack for $\mathrm{M}=0.85$. No buffet is indicated at $\mathrm{M}=0.70$.
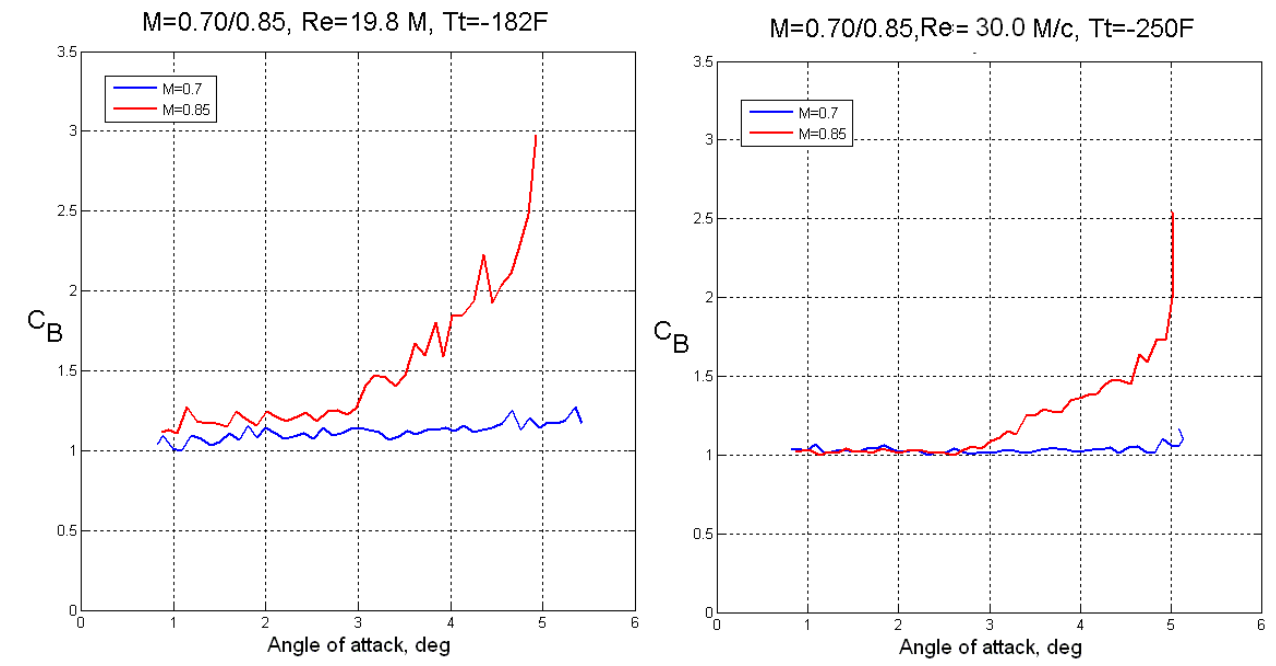

Figure 5: Buffet onset and intensity at High Reynolds number $R e=19.8$ and $30 \mathrm{M} / \mathrm{c}, \mathrm{M}=\mathbf{0 . 7 0}$ and 0.85 Wing Body Configuration without trip dots

Figure 6 shows that CRM left wing (top surface) and right wing (bottom surface) strain gauge dynamic data as $r m s$ strain. There is no qualitative difference except for amplitude. Since gauge locations are different for each wing, the amplitude difference is easily explained. Data shown is for $\mathrm{M}=0.70$ at Reynolds number of $5 \mathrm{M} / \mathrm{c}$. Similar behavior was found for all the runs at $\mathrm{M}=0.85$ and higher Reynolds numbers of 19.8 or $30 \mathrm{M} / \mathrm{c}$ for the left and right wing-root strain. There was a clear difference in static load data indicating a lift difference between the two wings, and the CRM developed a rolling moment once buffet onset occurred. 


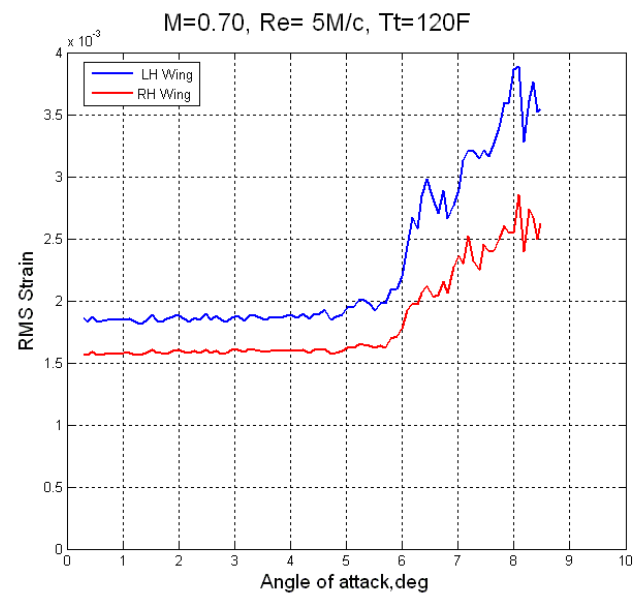

Figure 6: CRM Left/Right wings strain

Figure 7 shows all NTF M=0.85 data, covering both air mode and cryogenic testing at $R e=5,19.8$ and $30 \mathrm{M} / \mathrm{c}$ on a single plot. Despite differences in trip dot usage and a four to six-fold increase in Reynolds number, all data indicate onset of buffet at 3 degrees angle of attack.

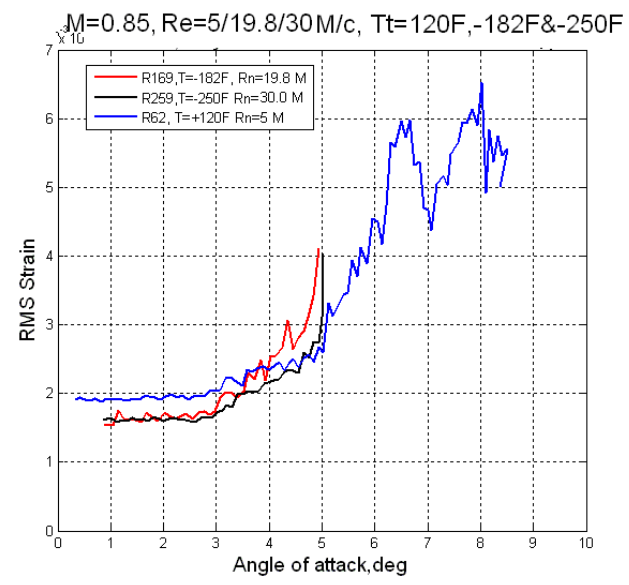

Figure 7: All $M=0.85$ data at $\mathrm{Re}=5,19.8,30 \mathrm{M} / \mathrm{chord}$

\section{CRM Kulite pressure analysis and correlation to buffet onset in NTF tests}

Figure 8 shows a signal schematic for the CRM Kulite responses. Kulites respond only to local pressure fluctuations and provide wideband pressure data as the output signal. As an angle of attack polar sweep is performed, flow separation is likely under high lift conditions. Kulites can pick up this separation only if it occurs right on the sensor surface. Similar to the case of strain gauge signals, the Kulite data was scaled to pressure units and then the spectrum was analyzed to arrive at $p_{\alpha}(f)$. Since the spectrum varies with angle of attack, a plot of the evolution of the spectrum for $\mathrm{M}=0.70$ and $\mathrm{Re}=5 \mathrm{M} / \mathrm{c}$ is shown in figure 9 . 


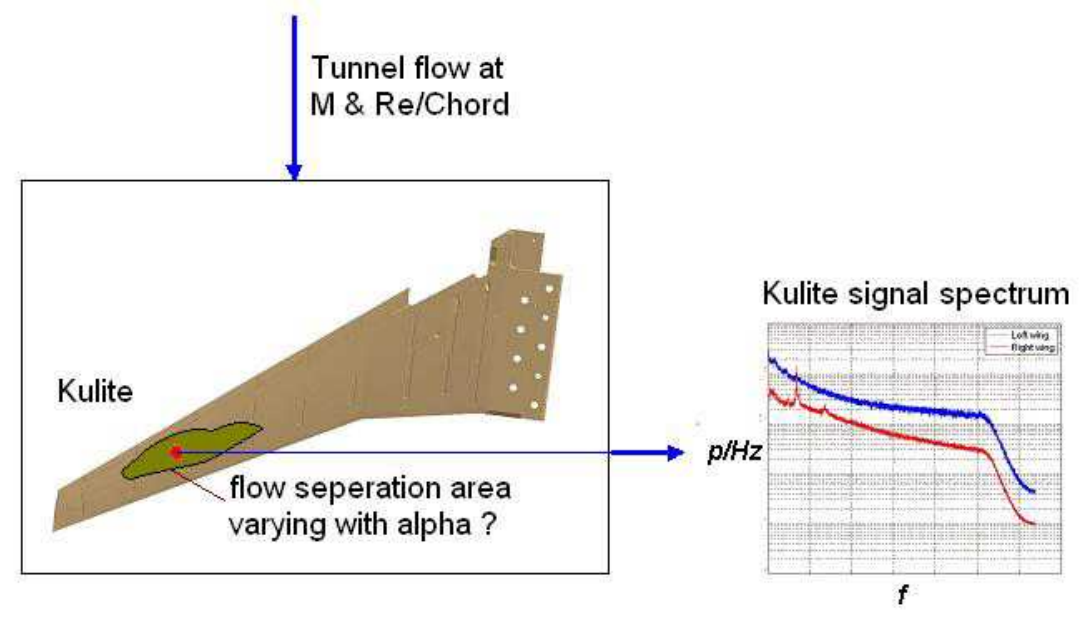

Figure 8: Kulite excitation and spectra

In figure 9, the left plot shows the raw Kulite signal, where $60 \mathrm{~Hz}$ noise effects are not apparent. The plot shows pressure amplitude growth correlated to angle of attack. The central plot shows pressure spectra for left and right wing Kulites. The spectra show that signal-to-noise ratio is good and $60 \mathrm{~Hz}$ noise was not an issue for NTF Kulites, as seen in the spectral plot. The left wing (top surface) clearly has higher fluctuations than the right wing (bottom surface). Pressure spikes at $710 \mathrm{~Hz}$ and its harmonics are due to Kulite cavity resonance and are not associated with any wing flow separation, as these occur at near zero angle of attack. The Kulite face was likely recessed from the wing surface during installation. Pressure spike data from all NTF air and cryogenic runs show the fundamental frequency of about 710,490 and $420 \mathrm{~Hz}$ followed by their harmonics. These occur at $120 \mathrm{~F},-182 \mathrm{~F}$ and $-250 \mathrm{~F}$ respectively, indicating that the frequency is related to square root of temperature and hence, the speed of sound. Thus, the data confirm that spikes are acoustic modes of the Kulite cavity. The plot on the right of figure 9 shows that as the angle of attack changes, an increase in pressure spectral amplitude is evident, indicating buffet intensity. Spikes occurring near zero angle of attack confirm the fact that cavity resonance is occurring in the Kulite well.
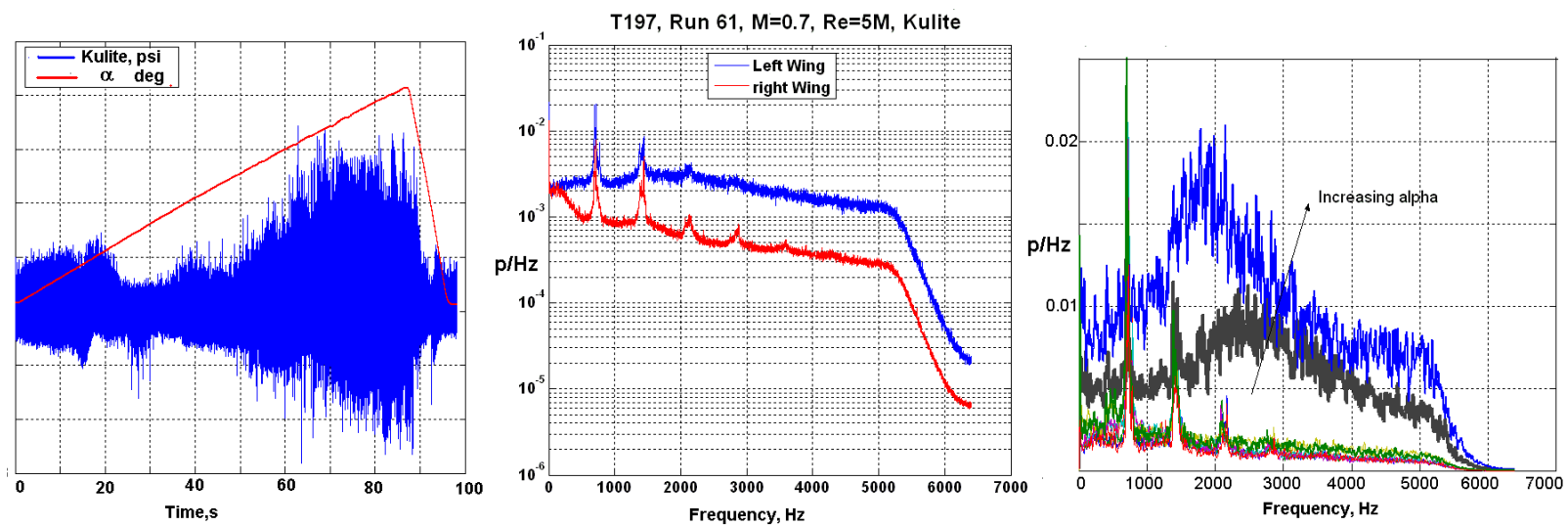

Figure 9: Kulite Pressure spectra and evolution with angle of attack

In figure $10, r m s$ Kulite pressure is shown as a function of angle of attack at $M=0.7 / 0.85$ and $R e=5 \mathrm{M} / \mathrm{c}$ flow. This data is generated by integrating $\mathrm{p}_{\alpha}$ spectral data. At $\mathrm{M}=0.7$, the top surface of the left wing sees a slow increase in Kulite pressure. No singular features are seen at 3 degrees angle of attack where the strain gauge data indicated buffet onset would start. This suggests that onset of flow separation seen by strain gauges did not occur at the Kulite location. However at 6 degrees where the buffet intensity increased rapidly (strain gauge data), a strong rise in $r m s$ Kulite pressure is seen suggesting that flow separation area is occurring over the Kulite location area from 6 degrees on. The bottom surface Kulite on right wing sees a weak increase above 6 degrees angle of attack. 

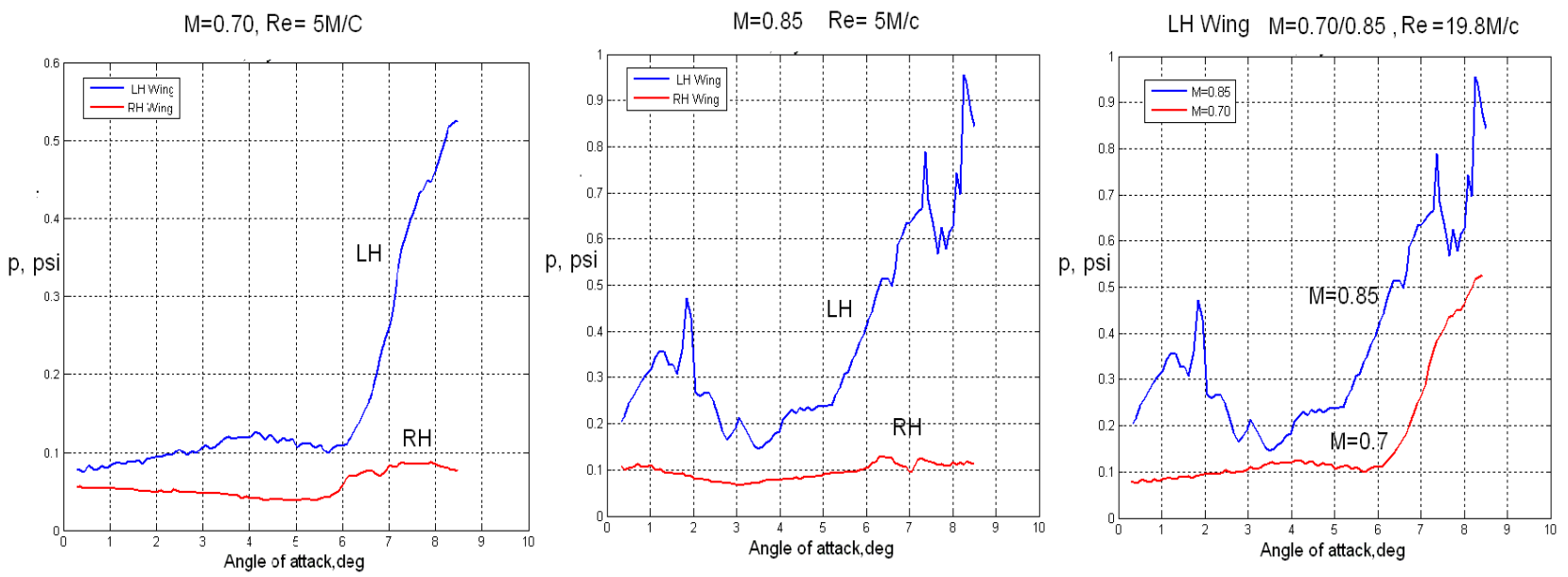

Figure 10: $M=0.7 / 0.85$, Reynolds number of $5 \mathrm{M} / \mathrm{c}$ Kulite pressure signature with angle of attack WB configuration, Trip dots $O N$

At $\mathrm{M}=0.85$ (in Fig. 10, plot 2), the top surface Kulite sees a sharp rise in pressure at 2 degrees angle of attack. This is not confirmed by strain gauge data. If anything, there is a minimum in rms Kulite pressure at 3 degrees angle of attack while strain gauge indicates onset of buffet. Clearly, this suggests that initiation of flow separation and buffet onset at 3 degrees angle of attack does not start at Kulite location. In Fig. 10, plot 3, left wing top rms Kulite pressures are compared for $\mathrm{M}=0.7$ and 0.85 .
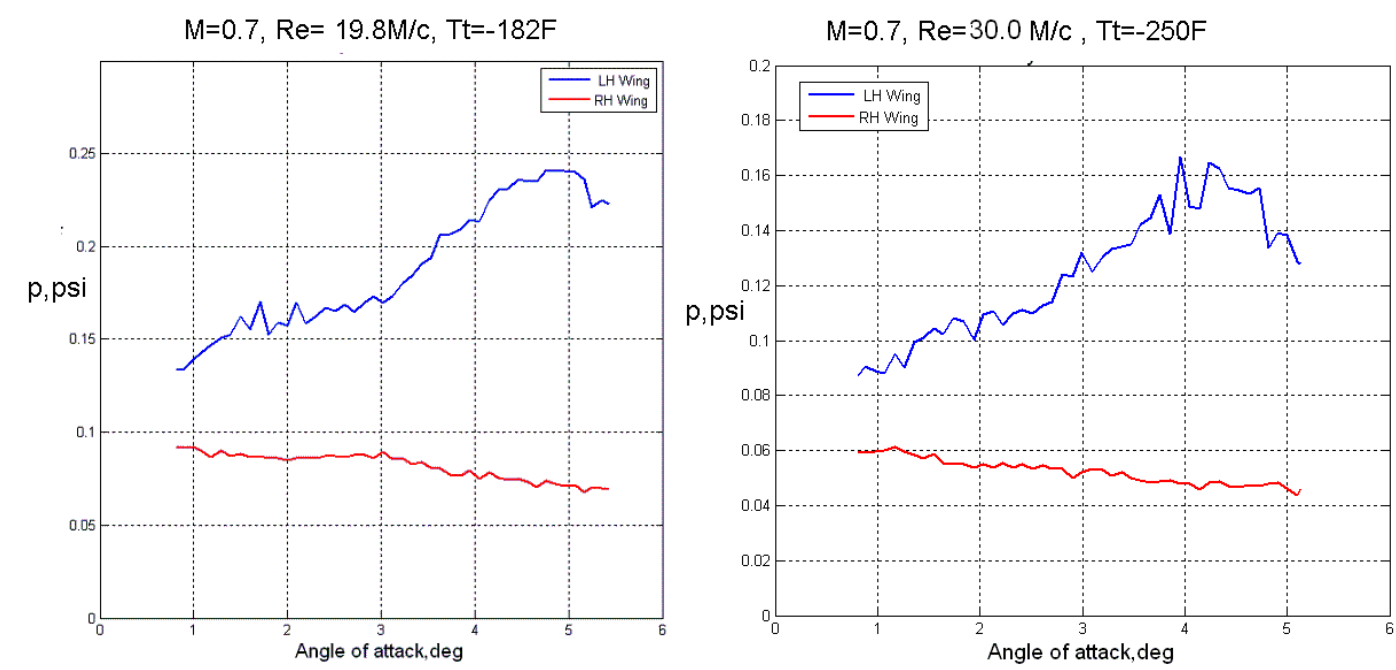

Figure 11: $M=0.7$, Reynolds number of $19.8 / 30 \mathrm{M} / \mathrm{c}$ Kulite pressure signature with angle of attack WB configuration, Trip dots OFF

In Figure 11, CRM Kulite pressure data for high Reynolds number runs at $\mathrm{M}=0.7$ are presented as a function of angle of attack. Kulite pressures show a monotonic increase in local fluctuations as a function of angle of attack with no singular features.

In Figure 12, CRM Kulite pressure data for high Reynolds number runs at $\mathrm{Re}=19.8 / 30 \mathrm{M} / \mathrm{c}$ and $\mathrm{M}=0.85$ are presented. In all $\mathrm{M}=0.85$ cases, left wing (top) Kulite data show a major pressure peak at about 3 degrees angle of attack. These data agree with the strain gage data in Figure 7 regarding the onset of buffet, pointing to flow separation occurring right over the Kulite location. The $-182^{\circ} \mathrm{F}$ runs show a larger Kulite peak compared to $-250^{\circ} \mathrm{F}$ and the reason for this is not clear. 

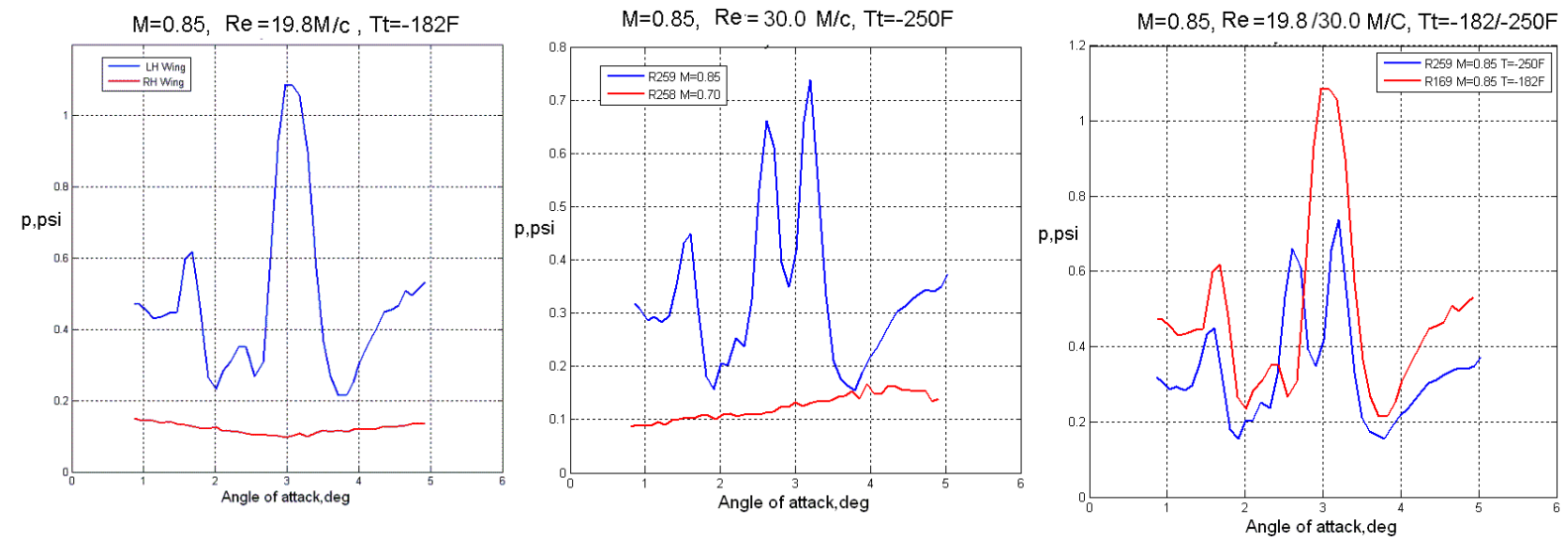

Figure 12: $\mathrm{M}=0.85$, Reynolds number of $19.8 / 30 \mathrm{M} / \mathrm{c}$ Kulite pressure signature with angle of attack WB configuration, Trip dots OFF

In summary, the Kulite rms pressure data from CRM tests shows that at $\mathrm{M}=0.7$, the Kulite does not see flow separation at its location until 6 degrees angle of attack. Further, during low Reynolds number runs $(\mathrm{Re}=5 \mathrm{M} / \mathrm{c})$ at $\mathrm{M}=0.85$, while wing-root bending moment data indicate onset of buffet at 3 degrees angle of attack, there is no correlation with Kulite rms pressure data. This suggests buffet onset at 3 degrees occurs due to flow separation initiation at locations other than the Kulite location. Above 6 degrees angle of attack, the Kulites do see a correlating $r m s$ pressure increase.

During high Reynolds number runs at $\mathrm{Re}=19.8 / 30 \mathrm{M} / \mathrm{c}$ and $\mathrm{M}=0.85$, wing-root gauges predict onset of buffet at 3 degrees angle of attack. The Kulite data confirm existence of strong pressure signatures near 3 degrees angle of attack at the Kulite location.

\section{Ames 11-foot TWT wing-root strain and Kulite data and comparison with NTF data}

The CRM model, balance and sting with dynamic instrumentation were moved to the 11-foot TWT and evaluated for force-moment-pressure, flow visualization with dynamic tests. The dynamic data was sampled at a rate of $12,800 \mathrm{~Hz}$. during. Figure 13, plot 1 shows a typical dual sweep polar of 0.4 degrees/s continuous pitch up to 8 degrees and returning to 0 degrees. Figure 13, plot 2 shows the Kulite pressure spectrum p/Hz. Figure 13, plot 3 shows the strain gauge signal spectrum, which had significant $60 \mathrm{~Hz}$ noise dwarfing the signals with a poor signalto-noise ratio relative to NTF tests. Though it was desirable to have very slow pitch rates, the slowest speed the 11foot TWT pitch system could provide was about 0.4 degrees/s. Angle of attack data was not sampled at same rate as the dynamic sensors creating problems with synchronizing $r m s$ strain gauge and Kulite dynamic data to $r m s$ angle of attack. However normal force (NF) was sampled at same rate and hence NF as percentile of full scale (FS) was used as an indirect measure of angle of attack. In analyzing NTF and 11-foot TWT wing root data, a spectral analysis time window width of 0.125 second and lower had to be used because of the faster pitch rate of 0.4 degrees $/ \mathrm{s}$ at 11 foot TWT and 0.8 degrees/s for NTF when pitching back down to 0 degrees. 


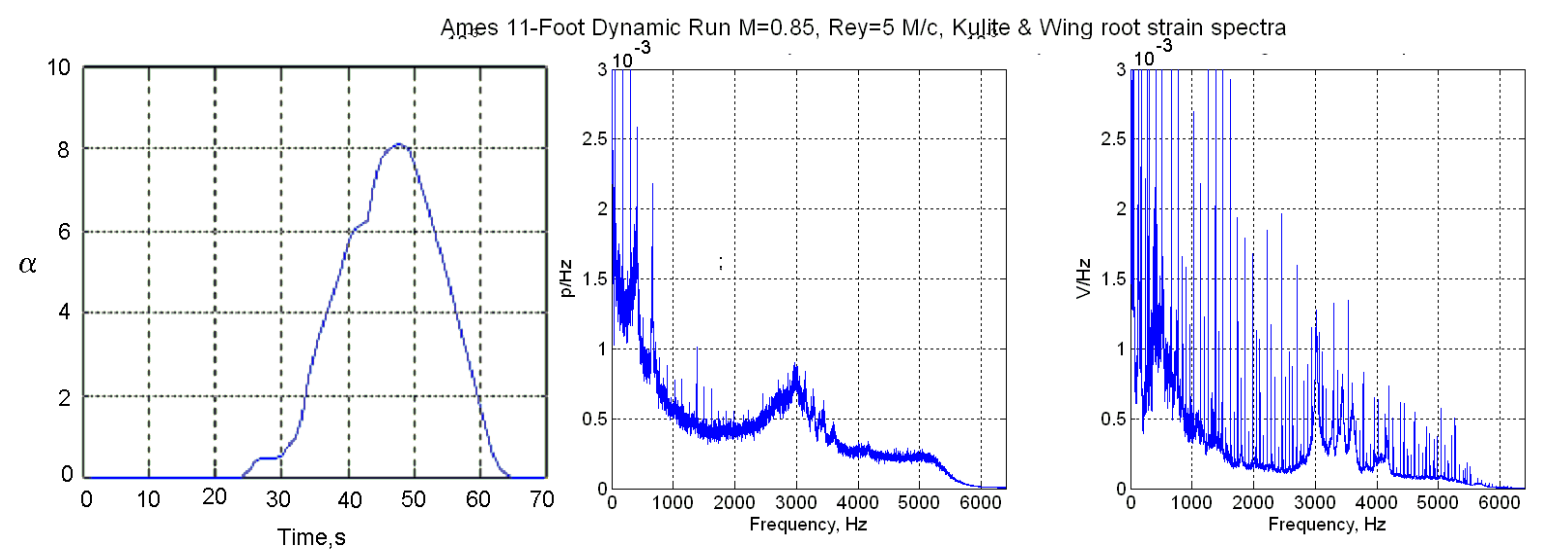

Figure 13: Ames 11-foot CRM test $\alpha$, Kulite Pressure and Wing root strain signatures

Figure 14 compares the wing-root strain gauge $\mathrm{S}_{\alpha}$ for $\mathrm{M}=0.7$ and 0.85 from NTF and 11-foot TWT. NTF wingroot strain shows a distinct change between $\mathrm{M}=0.7$ and $\mathrm{M}=0.85$ with buffet onset seen at about 3 degrees. In 11foot TWT data, $\mathrm{M}=0.70$ and $\mathrm{M}=0.85$ no such change was evident in $\mathrm{S}_{\alpha}$ except at high angles of attack. There is only single $\mathrm{S}_{\alpha}$ rise feature, and not two as in the NTF data. However, at $\mathrm{M}=0.87$, a signature of buffet onset at $32 \% \mathrm{NF}$ (corresponding to about 3 degrees angle of attack) is weakly visible with use of an expanded scale.
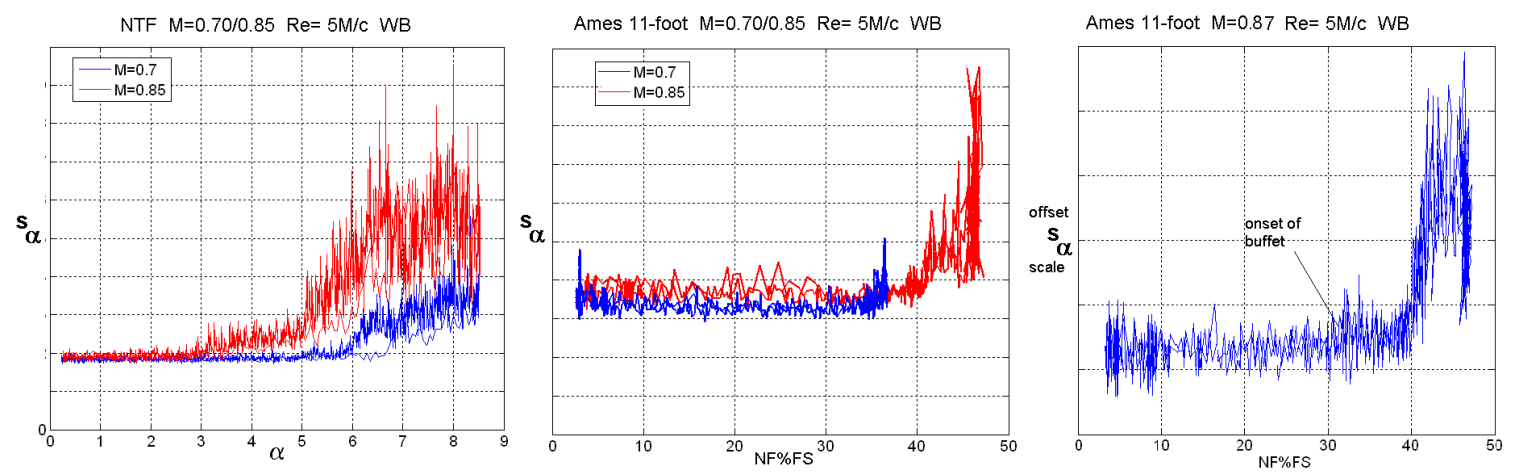

Figure 14: Wing-root strain $S_{\alpha}$ buffet onset responses from two facilities

Figure 15 shows data from $\mathrm{M}=0.85, \mathrm{Re}=5 \mathrm{M} / \mathrm{c}$ and Wing/Body configuration with trip dots from NTF and 11foot TWT dynamic tests. The left column plots compare angles of attack vs time during dynamic tests between the two facilities. Similarly, second column plots compare wing-root strain, $\mathrm{S}_{\alpha}$. Third column plots show Kulite pressure data, $\mathrm{P}_{\alpha}$. Use of a smaller time window provided a resolution of $+/-0.05$ degrees (and lower) in angle of attack associated with the rms Kulite pressure, rms wing-root strain or $r m s$ NF data. In these plots, the pitch sweep results are shown in blue lines and return pitch sweeps are shown in red.

In Figure 15, the NTF wing-root $\mathrm{S}_{\alpha}$ shows that buffet onset begins at 3 degrees angle of attack, in both sweep directions at different pitch rates of 0.1 degrees/s and 0.8 degrees/s. Faster return pitch rate data show slightly lower buffet intensity, but does not change the buffet signature at 3 degrees angle of attack or the higher buffet intensity for angles of attack greater than 6 degrees. However, wing-root $S_{\alpha}$ data from the 11-foot TWT show one buffet rise corresponding to about 6 degrees, and does not show the buffet signature at 3 degrees angle of attack seen in NTF data, except in $\mathrm{M}=0.87$ data. No major time delay issues are seen in the $\mathrm{S}_{\alpha}$ data when moving at fast or slow pitch rates at NTF. The reason as to why Ames 11-foot TWT $S_{\alpha}$ data does not explicitly show the buffet onset signature at 3 degrees is unclear, except to note that noise levels were very high in the signal. 
NTF $M=0.85, \operatorname{Re}=5 M / c$
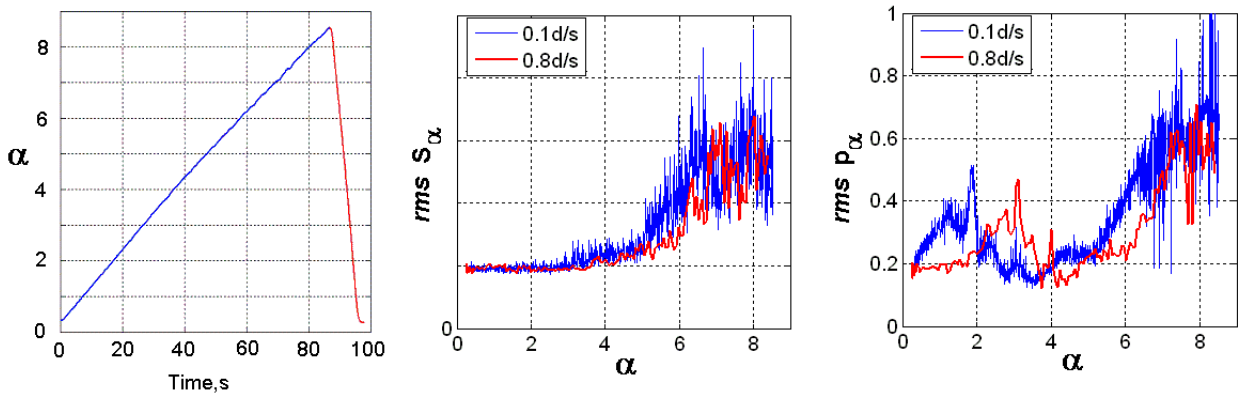

Ames 11-ft $M=0.85, \operatorname{Re}=5 M / c$
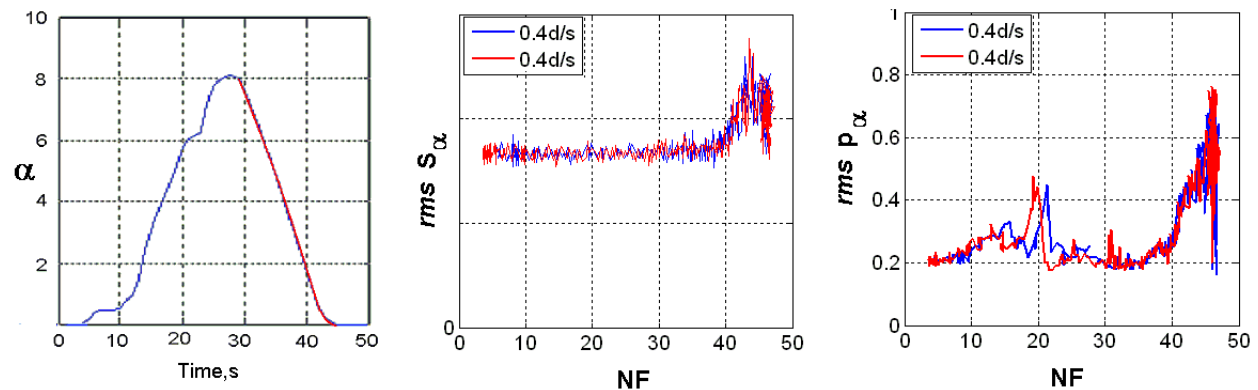

Figure 15: NTF and 11-foot TWT Wing root and Kulite signatures

In figure 15, the NTF Kulite data shows a shift in the $\mathrm{P}_{\alpha}$ peak occurrence between the slow forward sweep of 0.1 degrees/s and fast return sweep at 0.8 degrees/s. It appears Kulite events occur nearly 1 degree or about a second earlier during the faster return sweep. Clearly, NTF Kulite data is showing an unsteady aerodynamic feature in wing flow separation signature for the faster pitch rate. This unsteady aerodynamic effect is not strongly evident in the corresponding wing bending moment $\mathrm{S}_{\alpha}$. A possible explanation for the lack of unsteady effects in wing root strain $\mathrm{S}_{\alpha}$ data is that the full wing may have milder unsteady aerodynamics, thus washing out the relatively stronger unsteady effects seen at the Kulite location. Further, it also suggests that flow separation patterns have spatial and temporal features not all of which are seen at the chosen Kulite location. The 11-foot TWT $\mathrm{P}_{\alpha}$ data as a function of normal force show no major time delays in the Kulite signature, which is not surprising since the pitch rate is 0.4 degrees/s both in forward and return sweeps, and temporal effects are constant.

Wing root strain data, $S_{\alpha}$, results for the two transonic facilities could not be matched. NTF data clearly shows the initiation of buffet onset at 3 degrees angle of attack in a slow ramp and is repeated quite well in the faster return pitch data at $M=0.85$. There is no evidence of buffet onset at 3 degrees angle of attack in 11-foot TWT wing-root test data at $\mathrm{M}=0.85$.

\section{Summary of Results}

Dynamic tests on the CRM were intended to provide insight into model wing flow separation, buffet onset and their repeatability in different transonic test facilities. A continuous pitch rate was used to generate dynamic data by slowly increasing wing lift to the post-buffet zone. Flow separation effects were measured by wing-root sensors and Kulites.

\section{A. Wing-root strain gauge data}

1) Strain gauge half-bridge instrumentation was used for the CRM. With model internal wiring and further signal processing, $60 \mathrm{~Hz}$ noise levels were not very satisfactory at NTF. At 11-foot TWT the $60 \mathrm{~Hz}$ noise was even higher than for the NTF wing-root strain gauge noise levels.

2) Despite $60 \mathrm{~Hz}$ noise, NTF Wing-root strain bending moment data has provided reasonably consistent and repeatable buffet onset data as shown in Figures 4 and 14. 
3) Wing-root strain gauge data, $S_{\alpha}$, from the NTF could clearly identify the onset of buffet and flow separation as a function of angle of attack. Buffet onset is seen in the CRM at $\mathrm{M}=0.85$ consistently at 3 degrees angle of attack for $\mathrm{Re}=5,19.8$ and $30 \mathrm{M} / \mathrm{c}$ as shown in figure 7. Further, NTF data showed onset of buffet even during a 0.8 degrees/s fast return pitch movement, despite some unsteady lift features in the Kulites.

4) Ames 11-foot TWT wing-root strain data at $\mathrm{M}=0.85$ did not explicitly identify buffet onset at $32 \% \mathrm{NF}$. However, a weak signature was present at $\mathrm{M}=0.87$. It is certainly plausible that the weakness of signature could be due to signal noise.

5) The buffet intensity coefficient magnitude, $C_{B}$, is underestimated due to $60 \mathrm{~Hz}$ noise in the wing-root strain gauge data.

6) Wing-root sensors can not isolate the location of zones of flow separation over wings

7) Wing-root data, $S_{\alpha}$, is an integration of all separation effects on the full CRM wing area. $S_{\alpha}$ did not show major changes at faster pitch rates, due to unsteady lift aerodynamics.

\section{B. Dynamic pressure (Kulite) data}

1) The Kulite sensor is apparently recessed from the CRM wing surface, creating sharp cavity acoustic modes. Though undesirable, these cavity modes did not affect data quality. Also, Kulite cavity modes tended to disappear at higher angles of attack. This was evident in data from both test facilities.

2) NTF Kulite and wing-root strain gauge data for $\mathrm{M}=0.85$ and $\mathrm{Re}=19.8 / 30 \mathrm{M} / \mathrm{c}$ showed a correlation, suggesting that at the time of buffet onset, flow separation was present at the Kulite location.

3) The NTF Kulite pressure signatures at $\mathrm{M}=0.85$ and $\mathrm{Re}=5 \mathrm{M} / \mathrm{c}$ did not correlate very well with wing-root strain. This is probably because areas of initial flow separation over the wings do not occur at the Kulite location, but at other wing surface locations.

4) There was only a weak correlation for buffet onset in the 11-foot TWT Kulite fluctuating pressure wingroot strain data.

5) Kulite data vs angle of attack from NTF suggests that surface areas where flow separation occurs over the CRM wing varied with angle of attack, with temporal and spatial features, clearly moving in and out of the Kulite location area as seen in figure 12 .

6) Use of a fast pitch rate during dynamic testing clearly showed unsteady flow signatures as a time delay in the Kulite data, $\mathrm{P}_{\alpha}$, with respect to angle of attack. This effect was not shown in the wing root $\mathrm{S}_{\alpha}$ data dominantly or the buffet onset.

\section{Concluding remarks}

In contemporary wind tunnel work, balance signal quality receives singular attention with respect to noise, accuracy and, drift. However, dynamic sensors like wing-root strain gauge instrumentation and dynamic pressure instrumentation do not receive enough attention with regard to reducing electrical noise. To obtain accurate dynamic information like buffet onset, static mean load checks are not sufficient, since dynamic sensor instrumentation needs to have high signal-to-noise ratio over a wideband of frequencies. Further, there is need to perform dynamic testing at slow pitch rates to avoid issues of unsteady aerodynamics affecting results.

The analysis of CRM dynamic data has provided insight into the onset of buffet and growth of buffet intensity at $5,19.8$, and $30 \mathrm{M} / \mathrm{c}$ Reynolds numbers. At $\mathrm{M}=0.85$, the buffet onset for the CRM is at about 3 degrees angle of attack for 5, 19.8, and $30 \mathrm{M} / \mathrm{c}$ Reynolds numbers. Buffet intensity grows with increased angles of attack after buffet onset occurs. Wing-root strain gauge bridges provide repeatable data about wing buffet onset and possible flow separation over wings for the NTF data set. There are other definitions for buffet onset such as pitching-moment break or the normal-force break from polar aerodynamic data in published literature ${ }^{4}$. A quick check on aerodynamic polar data from NTF $M=0.85$ and $R e=5 \mathrm{M} / \mathrm{c}$ Wing/Body did show normal-force and pitching-moment breaks at 3.2 degrees, but no further study was made, as it was outside the scope of analysis of the CRM dynamic data. 


\section{Acknowledgments}

The NTF and 11-foot TWT CRM test teams provided extensive support in evaluation of the CRM dynamic testing work. Further, the authors wish to acknowledge encouragement and support from Melissa Rivers in the present work.

\section{References}

\footnotetext{
${ }^{1}$ Young, Clarence. P. Jr., Hergert, Dennis W., Butler, Thomas W., and Herring, Fred M., "Buffet Test in the National Transonic Facility," AIAA Paper 92-4032.

${ }^{2}$ Mabey, D.G., "Buffeting Criteria for a Systematic Series of Wings," Journal of Aircraft, Vol. 26, No. 6, June 1989.

${ }^{3} \mathrm{Su}$,Shen-Jwu., and Chow, Chuen-Yen., "Improvement of transonic Wing Buffet by Geometric Modifications," AIAA Paper 1993-3024.
}

${ }^{4}$ Clark, R.W., and Pelkman, R.A., "High Reynolds number testing of advance transport aircraft wings in National Transonic Facility,” AIAA Paper 2001-0910. 